\title{
e-Phaïstos
}

e-Phaïstos Revue d'histoire des techniques / Journal of the history of technology

IX-2 | 2021

Quel objet pour quel musée?

\section{Faire parler les objets}

Les collections dans les projets écomuséaux

Making Objects Talk: Collections in Ecomuseum Projects

\section{Claudia Da Re}

\section{(2) OpenEdition}

1 Journals

Édition électronique

URL : https://journals.openedition.org/ephaistos/9419

DOI : $10.4000 /$ ephaistos. 9419

ISSN : 2552-0741

Éditeur

IHMC - Institut d'histoire moderne et contemporaine (UMR 8066)

Référence électronique

Claudia Da Re, «Faire parler les objets », e-Phaïstos [En ligne], IX-2 | 2021, mis en ligne le 26 octobre 2021, consulté le 28 octobre 2021. URL : http://journals.openedition.org/ephaistos/9419; DOI : https://doi.org/10.4000/ephaistos.9419

Ce document a été généré automatiquement le 28 octobre 2021

Tous droits réservés 


\title{
Faire parler les objets
}

\author{
Les collections dans les projets écomuséaux
}

Making Objects Talk: Collections in Ecomuseum Projects

\section{Claudia Da Re}

1 À partir des années 1950, l'intérêt croissant des ethnographes et des muséologues pour le milieu industriel et urbain a mis en cause le modèle de musée connu jusque-là. La muséologie subit alors une révolution forte. Le musée cesse d'être considéré seulement comme un lieu de conservation et contemplation. Il devient un lieu avec une importante fonction éducative de masse. Parmi les causes de ce changement, se manifeste un intérêt croissant des musées d'ethnographie en faveur des milieux industriels et urbains ainsi que la nécessité de mieux définir le contexte qui incite à expérimenter les typologies d'implication directe du public. À côté des musées de plein air, d'autres typologies se développèrent, comme les Heimatmuseum, apparus en Allemagne entre les deux guerres mondiales. Ils avaient pour but de valoriser l'histoire d'une communauté ou l'œuvre d'un personnage local. Quant aux Folklife museums qui se répandent aux États-Unis à partir des années 1950, ils sont dédiés à la valorisation de l'histoire locale et à l'implication démocratique de la communauté, ou encore les Musées Atelier au Danemark où le public participe aux activités du musée. La réflexion autour du rôle du musée a avancé jusqu'à refuser l'idée du musée vu seulement comme lieu dédié à la conservation des objets, au profit d'un musée ouvert au territoire et plus attentif au rapport de l'homme à l'environnement. À ces grands bouleversements, il faut ajouter un facteur déterminant dans la naissance de la pensée écomuséale: l'insatisfaction et le désappointement d'un groupe de muséologues vis-à-vis de la dérive touristique-commerciale de beaucoup de musées traditionnels.

D'autre part, la réflexion muséologique a sûrement été touchée par des événements majeurs intervenus au cours de cette période :

- L'indépendance des anciennes colonies, suivie par les démarches de réaffirmation des identités locales ;

- Le mouvement pour l'égalité des minorités indiennes et afro-américaines en Amérique du Nord. 
De cette situation de crise émergent de nouveaux concepts à l'origine d'initiatives locales, comme celui d'écomusée, qui cherchent d'autres voies tout en maintenant leur attachement à la notion de patrimoine. Les écomusées se sont définis en grande partie contre le concept classique du musée-conservatoire d'objets, voire comme des musées sans collections.

\section{Nouvelle Muséologie}

4 Une réflexion initiale autour du musée apparaît déjà au $\mathrm{XVI}^{\mathrm{e}}$ siècle suivant les premières formes de classement et d'exposition des collections, comme les cabinets de curiosité. Mais son véritable développement commence durant le XIX ${ }^{\text {e }}$ siècle avec l'essor des musées, qui se différencient selon les collections, entre musées d'histoire, de folklore, musées de sciences et techniques, musées d'art, etc. L'étendue des typologies muséales détermine une diversification des parcours d'exposition, ainsi qu'une réflexion sur le rôle des musées dans la société. Bien que l'on publie déjà des écrits de muséologie au XIXe siècle, il faut attendre l'entre-deux-guerres et la création de l'Office international des musées (IOM) pour que ce mouvement trouve une légitimation internationale.

5 Les définitions qui ont été données au cours du temps sont nombreuses, mais à notre avis la plus complète est celle qui définit la muséologie comme « l'étude d'une relation spécifique entre l'homme et la réalité dont le musée, phénomène déterminé au cours du temps, ne constitue que l'une des concrétisations possibles » (Desvallées, Mairesse 2005 : 131-155). Cette définition tient compte des bouleversements théoriques survenus vers la fin des années 1960. À ce moment, l'institution muséale, conçue comme un lieu dédié à la collecte et à la conservation des objets, est remise en cause par une partie des muséologues et des experts qui lui reprochaient d'être trop éloignée du territoire. La décolonisation et la problématique de la restitution des biens culturels ont sûrement joué un rôle majeur dans cette question, favorisant l'introduction de concepts tels que la participation, la communauté et le territoire. La Table ronde de Santiago du Chili de 1972 a été déterminante en ce sens. Sa Déclaration finale a posé le rôle social du patrimoine culturel et donné une définition du musée intégral, fondé sur une connaissance interdisciplinaire et sur le rapport avec la communauté dont il doit promouvoir le développement. La déclaration affirme la suprématie de cette mission sur les fonctions traditionnelles du musée, conservation, bâtiment, objet, public. Elle peut être considérée comme le début d'un nouveau questionnement des musées et de leur rôle dans la société ; questionnement qui ne reste pas théorique, mais qui propose des solutions concrètes. On peut citer, dans sa lignée, l'écomusée du Creusot-Montceaules-Mines et l'écomusée de Marquèze, qui ont fait école.

6 Le Creusot a été un véritable laboratoire d'expérimentation autour des démarches participatives et sociales, où on a cherché à mettre en pratique les dispositions de la Déclaration de Santiago du Chili, réalisant notamment un écomusée communautaire (Corrias, Le Foll, Moëllo 2020). Au début des années 1980, toutes ces réflexions se concrétisent dans le mouvement de la Nouvelle Muséologie, qui trouve sa légitimation à l'échelle internationale avec la création du $\mathrm{MINOM}^{1}$ en 1985. Les motivations qui soustendent ce mouvement sont à rechercher dans le besoin de relâcher la rigidité du système et des principes sur lesquels s'appuyait jusqu'alors la muséologie. Mais le débat est plus large et prend aussi en considération le patrimoine. Les réflexions avancées par 
la Nouvelle Muséologie impliquent «l'homme tout entier, dans la nature tout entière, autrefois et maintenant, mais surtout à la recherche de son avenir et des instruments intellectuels et matériels de sa maîtrise " (Varine 1985 : 185). Dans une société qui intègre toutes les ressources du développement, la muséologie devrait chercher à s'ouvrir à de nouveaux champs d'intervention, au-delà de ses fonctions de collecte et de conservation, pour mieux intégrer son action dans l'environnement humain et physique (Déclaration de Québec).

7 La Nouvelle Muséologie se caractérise par une approche interdisciplinaire en considérant le développement des populations, mais sans oublier de préserver les objets qui témoignent d'une telle évolution. Ce qui change, ce n'est donc pas la façon de conserver les objets, mais plutôt l'approche qu'on a d'eux : l'objet exposé doit dialoguer avec le visiteur pour lui faire comprendre la relation à la réalité ; l'objet n'est pas conservé pour sa valeur en soit, mais en tant que représentation du contexte, de l'environnement et de la communauté dont le musée est le témoin. Dans cette vision, le monde entier devient patrimoine et donc tout est muséalisable. Le musée devient alors le lieu d'étude des relations de "l'homme à la réalité de l'univers dans sa totalité, et la muséologie est la science des relations de l'homme à la réalité de l'univers" (Desvallées, Mairesse 2011 : 30-37).

8 Les réflexions avancées par la Nouvelle Muséologie trouvent leurs racines dans les musées de voisinage, dans les musées scolaires, ainsi que dans les écomusées. Il nous semble pertinent d'affirmer que ces derniers ont été de véritables laboratoires d'expérimentation des théories de la Nouvelle Muséologie, à tel point que celle-ci est souvent assimilée à l'écomuséologie, ce qui n'est pas le cas. Alors que les premiers écomusées naissent de l'expérience d'ethnologues et muséologues dans le domaine des musées de société et de l'interaction avec des contextes ruraux et périphériques, les processus de redécouverte et de réévaluation de la dimension locale et de ses composantes, matériels et immatériels qui sont présents à la base de l'identité des lieux, sont parmi les principaux éléments distinctifs des écomusées.

\section{Écomusées : quelques définitions}

9 Les concepts d'écomusée et l'écomuséologie naissent en France dans les années 1970. Cette nouvelle conception muséale met au centre de ses réalisations l'homme et son environnement.

Le mot écomusée a été inventé un peu au hasard; Hugues de Varine l'explique très bien dans beaucoup de ses œuvres, y compris la dernière parue en 2017 (Varine 2017). L'histoire est connue : Hugues de Varine est en train d'organiser la Conférence Générale de l'ICOM qui doit se tenir à Paris et à Grenoble. L'arrêt nécessaire à Dijon devient l'occasion de faire intervenir Robert Poujade, le ministre de l'Environnement, qui est par ailleurs maire de Dijon. C'est lors d'un déjeuner entre Hugues de Varine, Georges Henri Rivière et Serge Antoine, le conseiller du ministre, que le mot écomusée est inventé. Le ministre Poujade l'utilisera pour la première fois lors du discours tenu pendant la Conférence ICOM et décidera ensuite de designer par ce mot tous les musées ou centres d'interprétation créés dans les Parc Naturels Régionaux. La première structure à prendre officiellement le nom d'écomusée fut le musée en plein-air de Marquèze, dans le Parc des Landes de Gascogne. 
11 Selon la définition donnée par Georges Henri Rivière ${ }^{2}$, l'écomusée consiste en une adaptation spécifique des musées de plein air. Il combine un musée du Temps, présentant l'histoire et l'identité du territoire concerné sous tous ses aspects et un musée de l'Espace, constitué par des sites répartis sur le territoire. Sa mission est d'enquêter sur la communauté dans son milieu. La participation de la population à la vie de l'écomusée est fondamentale pour la réussite du projet. Hugues de Varine ${ }^{3}$ radicalisera la définition de l'écomusée en 1973: "La communauté toute entière constitue un musée vivant dont le public se trouve en permanence à l'intérieur. Le musée n'a pas de visiteurs, il a des habitants » (Debary 2000 : 71-82). Selon Hugues de Varine, l'écomusée doit aborder la question de l'identité et la travailler avec la population, en lui proposant des outils d'interprétation critique.

On peut donc affirmer que les écomusées sont le résultat de la rencontre de deux démarches: la réflexion sur les musées de Georges Henri Rivière - qui trouve immédiatement un écho dans le public en raison de ses préoccupations essentielles : écologie et ethnologie régionale - et les aspirations participatives et autogestionnaires, qui ont conduit à envisager un nouveau type de musée (Hubert 1985 : 186-190). L'une des définitions les plus pertinentes reste celle originellement proposée par Georges Henri Rivière et Hugues de Varine. Elle fait référence aux différences entre musées traditionnels et écomusées.

\begin{tabular}{|l|l|}
\hline MUSEE & ECOMUSEE \\
\hline Collection & Patrimoine \\
\hline Bâtiment & Territoire \\
\hline Public & Communauté \\
\hline
\end{tabular}

Ce simple schéma met en évidence les éléments propres aux écomusées :

- Patrimoine : tout ce qui pour une communauté peut être considéré comme tel. La collecte, l'inventaire et l'interprétation du patrimoine se font grâce aux habitants qui contribuent à construire le patrimoine matériel et immatériel de l'écomusée à travers donations et prêts ;

- Territoire: il est vu comme le lieu des relations homme-nature, des savoirs des communautés locales. L'écomusée permet à la population de se réapproprier son propre territoire et prendre conscience de sa propre histoire ;

- Population : elle est le premier interlocuteur de l'écomusée et elle doit être impliquée dans chaque phase du projet écomuséal et territorial.

14 Selon Hugues de Varine, le concept d'écomusée reflète plusieurs idées qui peuvent être complémentaires entre elles :

- Son cadre de référence est le patrimoine d'une communauté ou bien son territoire ;

- Il dérive d'un long processus qui stimule le développement local ;

- La participation de la communauté est permanente et instrumentale à l'écomusée ;

- Il est un moyen de transmettre la culture locale, mais en même temps, l'écomusée est ouvert vers l'extérieur, dans une optique de partage des connaissances ;

- La recherche et la collecte des objets représentent des moyens pour réaliser la mission écomuséale, au lieu d'être les buts prioritaires de l'écomusée (Varine 2005). 
Amérique Latine et au Québec où il se mélange avec d'autres typologies similaires comme les Neighbourhood Museums ou les musées communautaires. L'Italie reste, jusqu'aux années 1990, en marge du débat. Elle continue de ne s'intéresser qu'au patrimoine culturel matériel. C'est la Région Piémont qui, la première, a créé des écomusées afin de sauvegarder le patrimoine traditionnel $\mathrm{du} \mathrm{XX}^{\mathrm{e}}$ siècle. Pour la doctrine italienne, l'écomusée est adossé à « une pratique participative de valorisation du patrimoine culturel, matériel et immatériel, élaborée et développée par un sujet organisé, expression d'une population locale, dans la perspective du développement soutenable ${ }^{4}$. L'idée italienne d'écomusée semble s'éloigner de celle qui constituait la volonté initiale de ces structures : naissance dans le contexte de critique du musée traditionnel, en se positionnant comme une alternative à lui et en donnant vie à une nouvelle forme, différente parce qu'ouverte et participative, diffuse et communautaire, mais de toute façon ancrée à une identité d'institution qui, dans le modèle italien, ne semble plus être considérable. En même temps, l'écomusée propose une vision globale et sans rupture entre l'homme et son territoire. Il incite la communauté à partager les activités.

17 Le but premier d'un écomusée réside dans la valorisation des différences entre nos sociétés rurale et métropolitaine, en mettant en exergue les caractéristiques, les richesses et les transformations. Un élément prépondérant dépend de la nature de l'initiative. Elle doit émaner de la communauté qui y est représentée. L'écomusée propose des solutions basées sur le souhait de la population, qui est toujours interpellée autour des différentes problématiques. L'écomusée n'est pas seulement une institution dévolue au bon fonctionnement d'un territoire. Il constitue plutôt une façon de concevoir le territoire et de faire face aux problèmes à travers la culture.

\section{Le rapport aux collections}

18 La volonté d'un renouvellement des musées passe aussi par une conception différente des collections. Normalement, quand le patrimoine culturel rentre dans les musées, il se trouve inséré dans des scénarios hors contexte. Selon Hugues de Varine, un travail de prise de conscience est nécessaire pour entamer un processus qui permet à l'homme de se transformer, par ses propres forces, d'objet en sujet. Cette théorie, rapportée au travail du musée, devient une double métaphore : «libérer les hommes par le musée revient à libérer les objets dans le musée » (Debary 2000 : 71-82). Les écomusées veulent favoriser une nouvelle représentation du patrimoine culturel en se plongeant dans le passé, non pas pour susciter de la nostalgie, mais pour aider à la compréhension des problèmes du présent. Leur structure interdisciplinaire et leur mission de diffusion des connaissances les portent, en quelque sorte, à s'engager dans les débats actuels sur le changement des structures de la société.

e-Phaïstos, IX-2 | 2021 
Le Code du Patrimoine considère comme étant un musée « toute collection permanente composée de biens dont la conservation et la présentation revêtent un intérêt public et organisée en vue de la connaissance, de l'éducation et du plaisir du public " (Art. L410-1). En revanche, dans les écomusées «l'ancien centre du musée - la collection - a été placé à la périphérie du système pour être remplacé par l'homme, vers qui l'institution se tourne. Celle-ci ne s'adresse pas au touriste de passage mais à l'individu qui vit sur le territoire dans lequel le musée a été installé. Ce territoire, cet environnement dans lequel des gens vivent et meurent, est la deuxième dimension dans laquelle s'inscrivent ces institutions " (Mairesse 2000 : 33-56). L'écomusée n'est donc pas simplement un conservatoire. Avec la participation de la population, il prend une part active à la vie de sa région, à son développement, en apportant sa contribution aux réflexions, aux débats et expérimentations. L'écomusée n'est même pas un lieu physique où garder le patrimoine culturel, il est plutôt le patrimoine.

Cette affirmation, qui peut apparaître un peu forcée, signifie qu'à la base de la création d'un écomusée apparaît la nécessité de sauvegarder le patrimoine local. Il faudrait alors élargir peut-être la signification de l'objet en l'étendant au territoire : l'objet à exposer et à mettre en valeur, ce sont les bâtiments, les activités commerciales, les fêtes, les habitants. Exposer non pas dans une optique de sensationnalisme folklorique, mais plutôt dans une optique de connaissance partagée, en interne avec les habitants et en externe avec les touristes. Une collection d'objet à proprement parler peut s'avérer un atout pour la compréhension du territoire et de sa population. Si elle est bien gérée et étudiée, cette collection rend possible la réalisation soit d'une exposition claire et cohérente, soit des recherches menées par des professionnels. Bien évidemment, la gestion de collections ne doit pas devenir l'activité principale de l'écomusée, étant donné que le but de ces structures n'est pas la conservation des objets. Un parcours d'exposition qui suit les règles muséographiques devient tout de même nécessaire, avec des panneaux explicatifs et une narration qui accompagne le visiteur (fig.1). À la différence d'un musée classique, dans l'écomusée, l'objet est inséré dans son contexte d'origine. Il dialogue avec ce dernier. 
Fig.1. Panneau explicatif du parcours de visite dans le village de Salbertrand, Italie

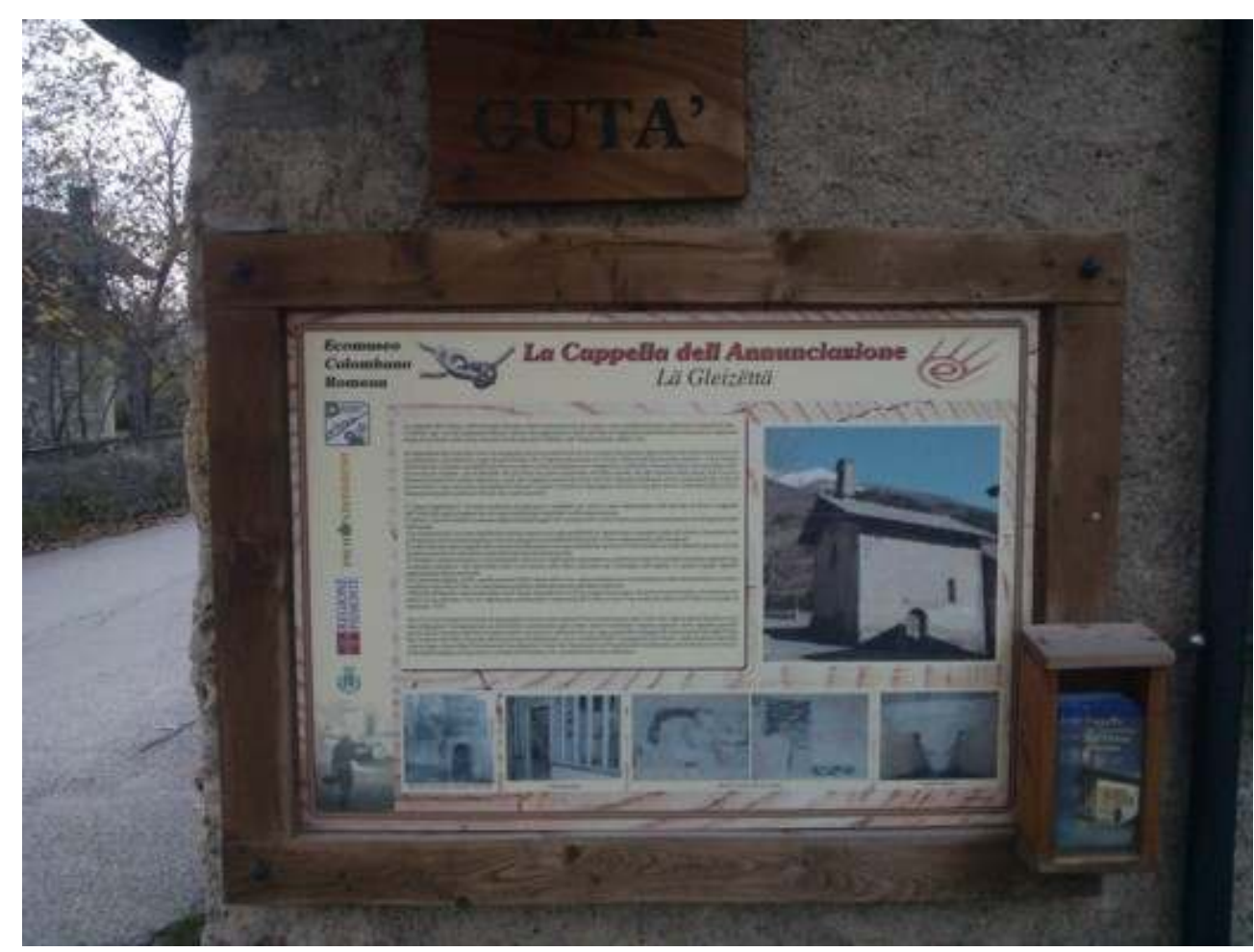

L'écomusée Colombano Romean se trouve dans le village de Salbertrand, dans la région du Piémont en Italie. II propose un itinéraire autour du village et du Parc du Gran Bosco pour découvrir l'histoire de ce territoire. L'image représente un des panneaux explicatifs que l'on peut trouver tout au long du parcours de visite.

Crédit : photo de l'auteur

Il est nécessaire d'utiliser les objets pour dialoguer avec la population. « La valorisation muséale porte non seulement sur le caractère historique de l'objet, mais aussi sur le geste de mémoire que constituent sa mise en public et sa patrimonialisation ». On sait que l'objet muséalisé, séparé de son contexte, a tendance à perdre son utilité d'origine et il en prend une autre, une utilité sociale dotée d'une signification particulière. Jean Davallon a décrit avec précision l'ensemble des gestes de la patrimonialisation après la rupture instaurée par la disparition du contexte: la « découverte de l'objet comme "trouvaille" ", la « certification de l'origine de l'objet », la « confirmation de l'existence du monde d'origine », la «représentation du monde d'origine par l'objet», la "célébration de la trouvaille de l'objet par son exposition", l'« obligation de transmettre aux générations futures» (Davallon 2011: 38-44). Dans les collections écomuséales, cela ne se produit pas très souvent. Grâce au travail de l'écomusée, l'objet garde ou retrouve sa signification d'origine. Il aide à reconstruire la mémoire d'un territoire. Autour des objets exposés, l'écomusée peut, ainsi, établir des activités pour faire découvrir aux nouvelles générations le patrimoine local, ou pour mettre en exergue les continuités et les ruptures des différentes générations.

Les collections écomuséales se constituent, d'habitude, grâce à des donations ou des dépôts d'objets par la population. Le catalogage et la création d'archives, ainsi que d'un lieu adapté, sont très importants pour la conservation des objets. Mais l'utilité d'une collection pour un écomusée réside dans son usage. L'écomusée forme un laboratoire où interroger les objets du patrimoine pour fournir à la population les instruments 
pour comprendre sa propre histoire. Pour cette raison, le patrimoine sauvegardé par un écomusée ne devrait pas se limiter à une époque précise, mais être toujours en constante création, parce que c'est la communauté elle-même qui le crée en vivant sur le territoire. Pour reprendre les mots de Bernard Schiele, le patrimoine constitue « un regard orienté. Un regard qualifiant un rapport au temps et à l'espace. On le conçoit naturellement tourné vers le passé. Ne pourrait-on pas - comme hypothèse de travail considérer qu'il est surtout interpellé par l'avenir, et que c'est en fonction de cela qu'il convoque le passé ?» (Schiele 2002 : 251). L'écomusée travaille justement dans ce sens, du moment que la collection, ainsi que les autres activités proposées, témoignent de l'existence d'un patrimoine culturel local, qui est étroitement lié aux vocations économiques du territoire, au paysage et aux changements survenus dans le temps. La prise en compte du passé est souvent une étape obligée dans la conception d'un développement futur qui se veut soutenable. En général, d'un point de vue muséologique, l'écomusée devrait contribuer à la formation du sentiment d'identité de la population; il devrait promouvoir des dynamiques sociales positives et favoriser l'amélioration de la qualité de vie des résidents.

C'est ici qu'émerge l'une des plus grandes difficultés pour un écomusée : celle de savoir dialoguer avec les nouvelles générations et les nouveaux résidents. La population se transforme au fil des temps et manifeste des besoins différents. L'écomusée doit donc réussir à accueillir cette nouvelle population pour qu'elle se sente représentée, sans pour autant oublier les générations précédentes. La pratique de l'inventaire participatif peut apparaître comme une solution très intéressante afin de déclencher cette prise de conscience de la part des habitants et aussi pour comprendre ce qui offre du sens pour eux : quels sont les lieux, les objets, les aspects que ces derniers veulent valoriser. Cette pratique peut prendre différentes orientations. L'Écomusée du Grand-Orly Seine Bièvre, par exemple, propose des expositions thématiques visant à mieux faire connaître un aspect du territoire et de la vie de la communauté. Dans ces conditions, les habitants sont invités à apporter des objets (cohérents avec la thématique en cours) qui sont catalogués et exposés (fig.2). Les objets peuvent ensuite rester à l'écomusée ou retourner chez leurs propriétaires, mais une trace écrite reste. Petit à petit, on prend conscience du patrimoine qui nous entoure. Une autre typologie d'inventaire participatif est représentée par les mappe di comunità ou parish maps (fig.3). Très utilisée par les écomusées italiens, cette pratique consiste à dessiner une carte du territoire sur laquelle est indiqué le patrimoine qui revêt du sens pour les personnes qui la réalisent. La construction de cette carte constitue tout à la fois un travail collectif et individuel qui aide les individus à découvrir ou redécouvrir leur propre histoire et à se réapproprier un paysage ${ }^{5}$. 
Fig.2. Exposition En-quête d'identités réalisée par l'Ecomusée du Grand-Orly Seine Bièvre en 2015

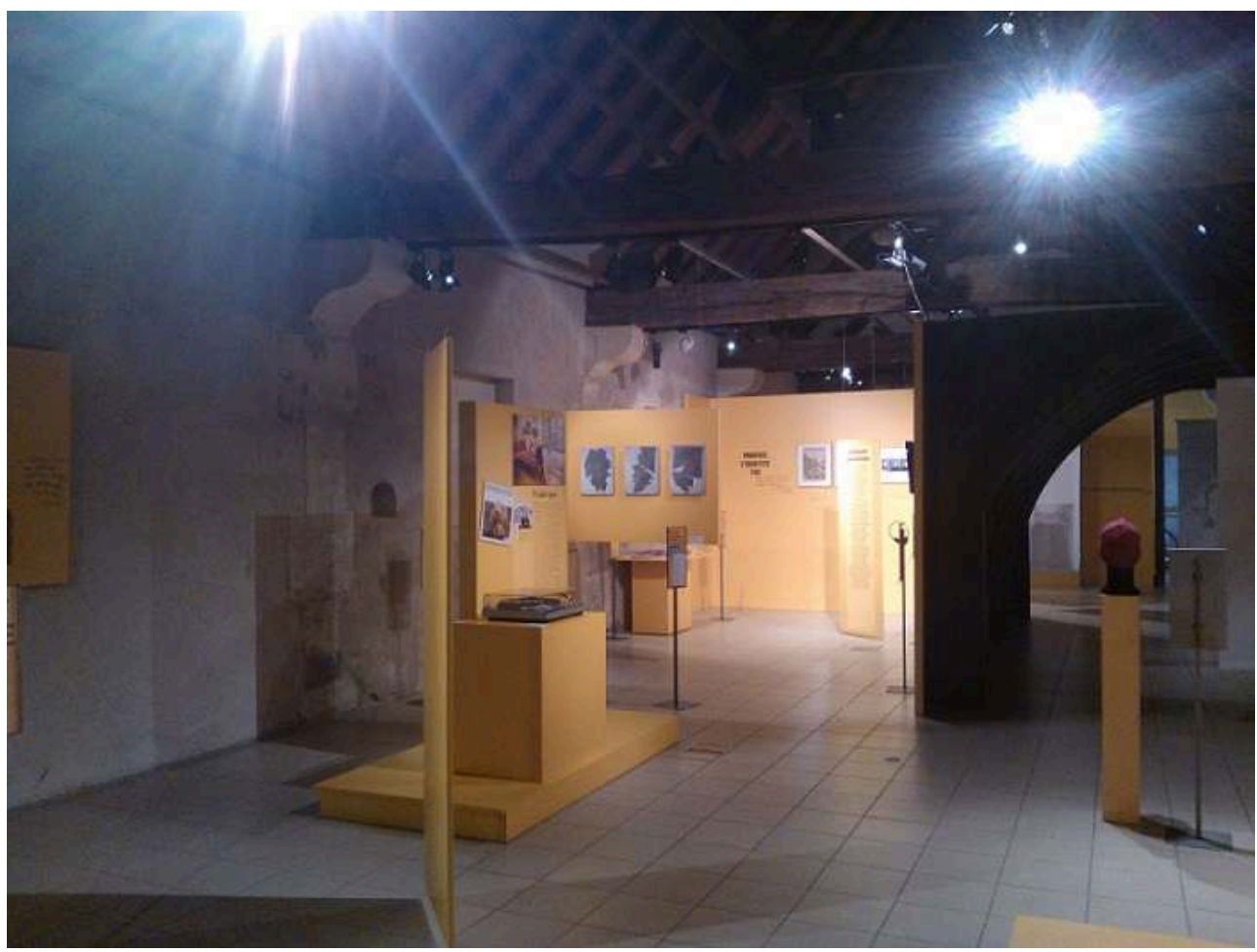

L'écomusée du Grand-Orly Seine Bièvre a toujours adopté une démarche participative dans ces activités, y compris les expositions. Les habitants sont régulièrement invités à apporter des objets et des témoignages afin d'enrichir la thématique de l'exposition. L'image montre une salle de l'exposition En-quête d'identités organisée du 3 avril 2015 au 31 janvier 2016 dans les salles de la Ferme de Cottinville. Dans ce cas, les habitants ont été interrogés autour de la notion d'identité individuelle et de groupe.

Crédit : photo de l'auteur 
Fig.3. Parish maps de l'Ecomusée Colombano Romean de Salbertrand, Italie

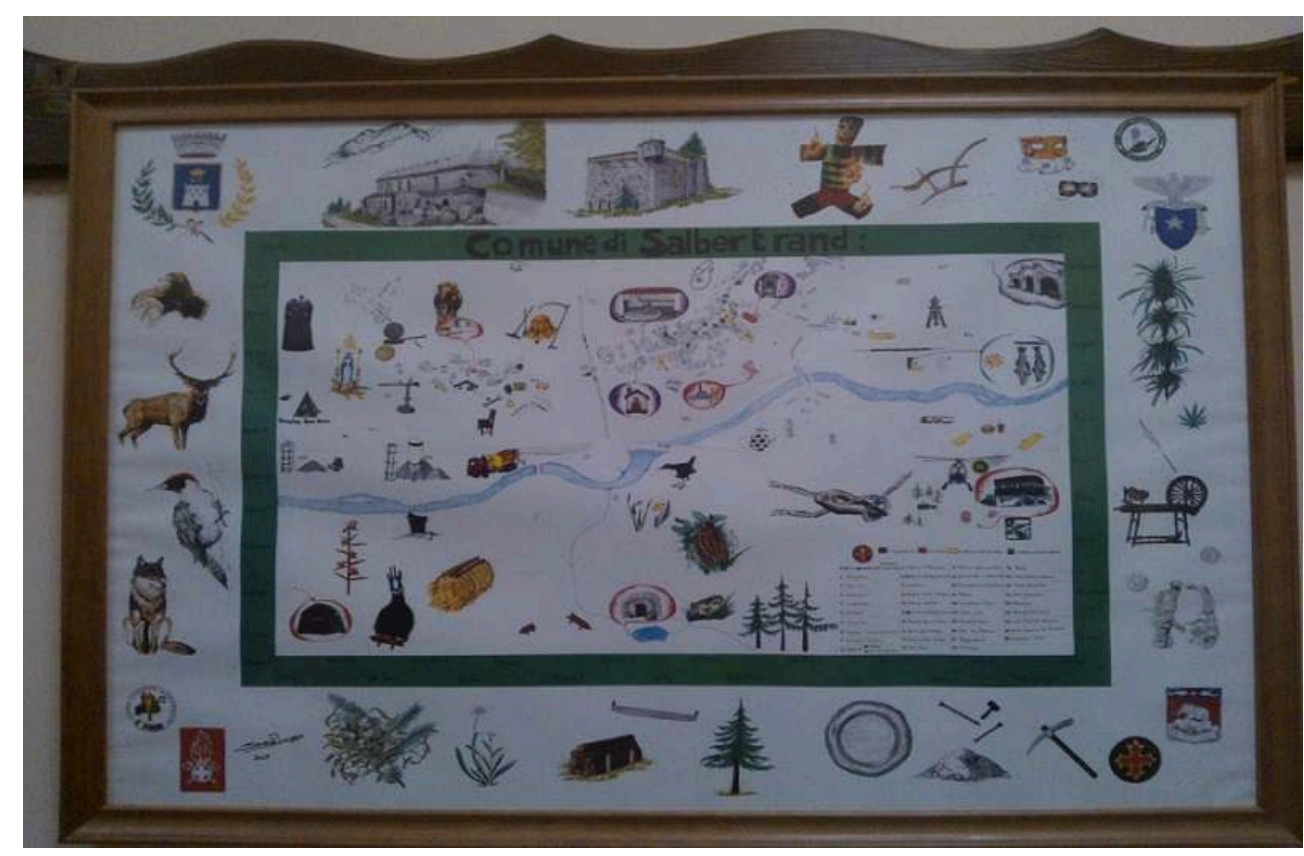

L'écomusée a réalisé sa propre parish maps avec la participation deux chercheurs de la Faculté d'anthropologie de l'Université de Turin. Les habitants ont été orientés vers un processus de réappropriation du territoire qui a donné comme résultat la parish map. Ce document témoigne de la perception que les habitants ont de leur propre territoire.

Crédit : photo de l'auteur

Pour que l'inventaire participatif soit un instrument pour implémenter les missions de l'écomusée, il faudrait le renouveler régulièrement car la perception de ce qui est patrimoine change au cours du temps et avec les générations. Souvenons-nous que le patrimoine qui est reconnu dans ce contexte est celui dans lequel la population s'identifie et à travers lequel elle se sent représentée. Cette démarche d'utilisation du patrimoine comme outil d'action sociale et citoyenne donne aux habitants la maitrise de leur propre patrimoine considéré dans son évolution et aide à renforcer l'identité locale puisqu'elle cherche à rendre la population plus consciente de l'importance de s'engager pour son territoire. C'est pour cela que l'on dit que les écomusées doivent représenter le produit d'une négociation entre les différents membres de la communauté et non pas la simple exposition de l'espace anthropique. Ils doivent rendre transparents les procès de réappropriation de la mémoire collective qui ont déterminé sa propre constitution. Ils doivent aussi insister sur les valeurs qui soustendent leur réalisation. Le devoir d'un écomusée est alors de créer des moments de dialogue avec la population, en cherchant à l'impliquer activement dans la gestion et dans l'organisation des activités, en développant ou en redécouvrant le sens d'appartenance à un territoire et à une communauté.

\section{Conclusion}

Le désir de renouvellement qui était à la base de la naissance des écomusées, vers la fin des années 1960, a entraîné ces structures à se positionner par rapport aux musées traditionnels. La collection, l'objet étant les éléments fondateurs de ces derniers, ont été toute de suite remis en cause. La condamnation totale du modèle muséal connu 
jusque-là a véhiculé l'idée que l'écomusée est un lieu dépourvu de collections, et cela d'autant plus que l'éventuelle présence d'une collection aurait limité l'activité d'un écomusée. Or, on a bien vu qu'éviter de collecter des objets est presque impossible : les donations ou l'envie de sauver de la destruction des mobiliers, des machines ou parfois des bâtiments, ont fait que, peu à peu, tous les écomusées se sont retrouvés à gérer des collections. Ce sont même souvent les objets réunis par les écomusées qui garantissent le maintien de leur activité.

En même temps, le rapport aux éléments de la tradition muséale a changé et la collecte des objets n'est plus perçue comme un obstacle. Certes, quelques écomusées en France ont évolué vers une structure plus classique. Ils répondent mieux aux orientations de la Loi sur les Musées qui encadre toutes les institutions muséales confondues. Mais en proposant un discours plus général, tenant compte d'une réalité locale ou nationale, italienne par exemple, la présence d'objets peut devenir un atout, un instrument supplémentaire en vue d'interroger le patrimoine et la communauté.

La présence d'un parcours d'exposition aide les visiteurs à se plonger dans la réalité du territoire et à mieux comprendre l'évolution du paysage et, de manière plus générale, la culture du lieu qu'ils sont en train de visiter. La collection a aussi une autre fonction, peut être encore plus importante. Comme on a cherché à le démontrer dans cet article, les objets collectés peuvent stimuler ou enrichir des démarches participatives. Cela constitue, à notre avis, un point fondamental. En effet, c'est à travers ce genre d'activités que l'on découvre notre histoire et que l'on prend conscience de l'existence d'un patrimoine culturel, qui n'aura pas une valeur universelle certes, mais une valeur pour nous-mêmes, nous représentant en tant que communauté, et parfois en tant qu'individu.

\section{BIBLIOGRAPHIE}

BALLÉ Catherine, « Musées, changement et organisation », Culture \& Musées, n², 2003, p.17-33 ; DOI : https://doi.org/10.3406/pumus.2003.1176

CORRIAS Pauline, LE FOLL Typhaine, MOËLLO Morgane, « L’Écomusée Creusot Montceau : métaphore d'un modèle exemplaire », e-Phaïstos [En ligne], VIII-1 | 2020, mis en ligne le 29 avril 2020, consulté le 19 septembre 2021 ; DOI : https://doi.org/10.4000/ephaistos.7679

DA RE Claudia, «Increase the Potential of a Territory Starting from Culture. The Exemplar of the Ecomuseo della Pastorizia », in PINTON Simona, ZAGATO Lauso (eds) Cultural Heritage. Scenarios 2015-2017, Venise, Cà Foscari Edizioni, 2017, p.639-656; URL : https://edizionicafoscari.unive.it/ media/pdf/books/978-88-6969-179-9/978-88-6969-179-9_CGTicPb.pdf

DAL SANTO Raul, STEFANI Adriana, BALDI Nerina, DEL DUCA Andrea, ROSSI Andrea, « The strategic Manifesto of Italian Eco-museums: Aims and Results », in RIVA Raffaella (ed.), Ecomuseums and Cultural Landscapes. States of the Art and Future Prospects, Santarcangelo di Romagna, Maggioli Editore, 2017, p.351-363; URL : http://www.sitda.net/downloads/biblioteca/ Riva_Ecomuseums_ebook.pdf 
DAVALLON Jean, «Le pouvoir sémiotique de l'espace. Vers une nouvelle conception de l'exposition ? ", Hermès, La Revue, 2011, vol.3, n61, p.38-44 ; DOI : https://doi.org/10.3917/herm. 061.0038

DEBARY Octave, «L'écomusée est mort, vive le musée », Culture \& Musées, n¹7-18, 2000, p.71-82 ; DOI : https://doi.org/10.3406/pumus.2000.1156

DELARGE Alexandre (dir.), Le Musée participatif. L'ambition des écomusées, Collection « MuséesMondes ", Paris, La Documentation française, 2018

DESVALLÉES André, MAIRESSE François, « L'organisation des musées : une évolution difficile », Hermès, La Revue 2011, vol.3, n61, p.30-37 ; DOI : https://doi.org/10.3917/herm.061.0030

DESVALLÉES André, MAIRESSE François, «Sur la muséologie », Culture \& Musées, n6, 2005, p. 131-155 ; DOI : https://doi.org/10.3406/pumus.2005.1377

GRASSENI Cristina (ed.), « Ecomuseologie. Pratiche e interpretazioni del patrimonio locale », Quaderni des CE.R.C.O., ${ }^{\circ}$ 6, Rimini, Guaraldi, 2010 ; URL : https://iris.unito.it/retrieve/handle/ 2318/63170/7825/Ecomuseologie_pdf00673.pdf

HUBERT François, « Les écomusées en France : contradictions et déviations », Museum, $\mathrm{n}^{\circ} 148$, (vol. XXXVII, n4 1985), p.186-190 ; URL : http://www.ecomusei.eu/mondilocali/wp-content/ uploads/2011/03/1985-Museum-127347b.pdf

MAIRESSE François, « La belle histoire, aux origines de la nouvelle muséologie », Culture \& Musées, n¹7-18, 2000, p.33-56 ; DOI : https://doi.org/10.3406/pumus.2000.1154

POULOT Dominique, «Introduction », Culture \& Musées, n8, 2006, p.13-25 ; DOI : https://doi.org/ 10.3406/pumus.2006.1402

SCHIELE Bernard, Patrimoines et identités, Québec, MultiMondes Éditions, 2002

TESTA Ilaria, MURTAS Donatella, « Mappe di comunità : storie di uomini e di luoghi », Ecomusei.net (portale della Regione Piemonte) ; URL : http://www.ecomusei.net/mappe-dicomunita

VARINE Hugues de, «L'écomusée : au-delà du mot ", Museum, n¹48 (vol. XXXVII, n4, 1985), p. 185 ; URL : http://www.ecomusei.eu/mondilocali/wp-content/uploads/2011/03/1985Museum-127347b.pdf

VARINE Hugues de, Les racines du futur : Le patrimoine au service du développement local, Paris, ASDIC Éditions, 2005

VARINE Hugues de, «Le musée, agent et acteur de la soutenabilité du développement des territoires ", Intervention au séminaire Musées et développement durable, Université de Bourgogne, 4 et 5 mars 2010, Dijon, www.hugues-devarine.eu.

VARINE Hugues de, L'écomusée singulier et pluriel. Un témoignage sur cinquante ans de muséologie communautaire dans le monde, Paris, L'Harmattan, 2017

\section{NOTES}

1. Mouvement International pour une Nouvelle Muséologie (MINOM), créé en 1985 à Lisbonne, pendant le deuxième Workshop International sur la Nouvelle Muséologie. Il rassemble des professionnels des musées communautaires, écomusées, écoles de muséologie, de gestion et de médiation culturelle et des institutions muséales ayant des relations interactives avec les 
collectivités locales. Le MINOM est un organisme affilié à l'ICOM ; URL : http://www.minomicom.net .

2. Georges Henri Rivière (1897-1985) a été un important muséologue et le fondateur du Musée des Arts et Traditions Populaires, qui est devenu le MUCEM de Marseille. Son œuvre beaucoup apporté à la Nouvelle Muséologie et au milieu des musées ethnographiques. Il est l'un des concepteurs de la notion d'écomusée.

3. Archéologue, historien et muséologue, Hugues de Varine a dirigé l'ICOM de 1965 à 1975. C'est, avec G.-H. Rivière, l'autre principal contributeur de la Nouvelle Muséologie et des écomusées dont il s'occupe toujours.

4. Conférence Giornate dell'ecomuseo_Verso una nuova offerta culturale per lo sviluppo sostenibile del territorio. Elle s'est tenue à Catane, en Sicile, les 12 et 13 octobre 2007.

5. Voir Testa, Murtas, nd.

\section{RÉSUMÉS}

L'article se propose de réfléchir sur l'existence des collections dans les écomusées. En faisant tout d'abord une introduction sur l'évolution des concepts de la muséologie et sur ce qu'il faut entendre par écomusée, on verra que le rapport à l'objet a toujours été pris en compte. L'écomusée est une façon de concevoir le territoire et de faire face aux problèmes à travers la culture. L'écomusée travaille justement dans ce sens, du moment que la collection, ainsi que les autres activités proposées, témoignent de l'existence d'un patrimoine culturel local, qui est étroitement lié aux vocations économiques du territoire, au paysage et aux changements survenus dans le temps.

This paper aims to reflect about the presence of collections in ecomuseums. We will start with a brief introduction on the evolution of museums studies and ecomuseology, which will show us that the relationship between objects and ecomuseums it has being considered since the beginning. The ecomuseum approach focuses on the recreation of territorial knowledge, which is based on the interconnection of know-hows and on the promotion of cultural heritage by individuals who reinterpret them as a collective resource. Using cultural heritage as an instrument of social and democratic action gives people the tools to understand their own cultural heritage, considered in its evolution, and helps to strengthen the local identity by increasing the population's awareness of the importance of taking action to safeguard its territory.

\section{INDEX}

Mots-clés : histoire des techniques, écomusée, patrimoine culturel, patrimoine immatériel, soutenabilité

Keywords : history of technology, ecomuseum, intangible heritage, cultural heritage, sustainability 


\section{AUTEUR}

\section{CLAUDIA DA RE}

Claudia Da Re est ingénieur d'études au sein de l'Université Gustave Eiffel dans le laboratoire DICEN-IdF. Ses thématiques de recherche concernent la problématique de la soutenabilité culturelle et les politiques de sauvegarde du paysage. Elle prépare une thèse en Histoire des techniques qui porte sur l'étude comparative de l'histoire des écomusées entre la France et l'Italie. Dans le cadre de ses recherches, elle s'intéresse à la notion de patrimoine, ainsi qu'au rapport des populations avec leur milieu au sens anthropologique du terme. 